\title{
Concordancia en la estimación del consumo máximo de oxígeno entre una prueba de esfuerzo y el Polar s810®
} Agreement between a fitness test and Polar S810 for estimating maximum oxygen uptake

\author{
Edgar Cortés-Reyes ${ }^{1}$, Jairo Echeverry-Raad ${ }^{2}$, Erica M. Mancera-Soto ${ }^{1}$ y
} Diana M. Ramos Caballero 3

\begin{abstract}
1 Departamento del Movimiento Corporal Humano, Facultad de Medicina; Universidad Nacional de Colombia, Bogotá, D.C. ecortesr@unal.edu.co, emmanceras@unal.edu.co

2 Facultad de Medicina; Universidad Nacional de Colombia, Bogotá, D.C. jecheverryr@unal.edu.co 3 Escuela de Medicina y Ciencias de la Salud, Universidad del Rosario. Bogotá, Colombia diana.ramos98@urosario.edu.co
\end{abstract}

Recibido 19 marzo 2009/Enviado para Modificación 8 Septiembre 2009/Aceptado 17 Septiembre 2009

\section{RESUMEN}

Objetivo Identificar la concordancia entre los datos de estimación del consumo máximo de oxígeno, obtenidos mediante una prueba de esfuerzo y el monitor de frecuencia cardiaca Polar S810®.

Métodos Se hizo un estudio observacional de concordancia entre dos métodos de estimación del $\mathrm{VO}_{2 \max }$, en forma simultánea e independiente. Se aplicaron dos protocolos, uno en reposo y otro en esfuerzo máximo, en 46 deportistas de la Universidad Nacional de Colombia, entrenados en deportes de resistencia aeróbica. Resultados El Coeficiente de correlación-concordancia (CCC) de Lin fue 0,31 ( $p<$ $0,003)$. Este coeficiente no se modificó sustancialmente al estratificar por género (masculino: CCC 0,29 IC $95 \%$ 0,04 - 0,54; femenino CCC 0,15 IC $95 \%-0,14-0,45$ ), por tipo de deporte (grupal: CCC 0,38 IC $95 \%$ 0,04 - 0,54; individual: (CCC 0,16 IC 95 $\%-0,20-0,52)$ y tan solo en los deportistas entre 24,1 y 28 años de edad mejoró su valor (CCC 0,65 IC $95 \%$ 0,35-0,93). Teniendo como referencia la prueba de esfuerzo, el Polar $\mathrm{S} 810 \AA$, da valores superiores o inferiores, contando como punto de corte los $42 \mathrm{~mL} / \mathrm{Kg} / \mathrm{min}$.

Conclusiones La concordancia entre los dos métodos fue pobre en forma general y en los diferentes subgrupos de deportistas, lo cual no permite intercambiar los resultados de estimación del $\mathrm{VO}_{2 \max }$ obtenidos entre la prueba de esfuerzo y el Polar S810®.

Palabras Clave: Prueba de esfuerzo, frecuencia cardiaca, ejercicio, consumo de oxígeno (fuente: DeCS, BIREME)

\section{ABSTRACT}

Objective Identifying agreement between data for estimating maximum oxygen uptake obtained by a fitness test and the Polar S810 heart rate monitor. 
Methods This was an observational agreement study comparing two methods for estimating $\mathrm{VO}_{2 \max }$ (simultaneously and independently). Two protocols were applied to 46 athletes from the Universidad Nacional de Colombia who had been trained in endurance sports (one at rest and maximum effort i).

Results Lin's concordance correlation coefficient CCC) was $0.31(p<0.003)$. This coefficient did not change substantially when stratified by gender (male: CCC 0.29 ; 0.04 $0.5495 \% \mathrm{Cl}$; female CCC 0.15; 0.14-0.45 $95 \% \mathrm{Cl}$ ), type of sport (group: CCC 0.38; $0.04-0.5495 \% \mathrm{Cl}$; individual: CCC $0.16 ; 0.20-0.5295 \% \mathrm{Cl}$ ) and its value only improved in athletes aged 24.1 to 28 (CCC $0.65 ; 0.35-0.9395 \% \mathrm{Cl}$ ). Regarding the maximum test, the Polar S810 gave higher or lower values, with $42 \mathrm{~mL} / \mathrm{kg} / \mathrm{min}$ cut-off.

Conclusions Agreement between both methods was generally poor and in the different subgroups of athletes; this meant that the results obtained from estimating VO2max could not be exchanged between the fitness and Polar S810 tests.

Key Words: Fitness test, heart rate, exercise, oxygen consumption (source: $\mathrm{MeSH}$, NLM).

$\mathrm{E}$ 1 deporte es una estrategia de promoción de estilos de vida saludables y buen uso del tiempo libre y se constituye en un derecho fundamental de las personas, contribuyendo a su desarrollo integral (1). Es una herramienta disponible y costo-efectiva para promover, prevenir y mejorar condiciones de salud a lo largo del ciclo vital humano. Sin embargo, no siempre contempla medidas evaluativas que permitan hacerlo una práctica segura, pues eventualmente podría generar riesgos, tales como sobre entrenamiento, fatiga, muerte súbita, entre otros (2). No siempre se tiene conocimiento y acceso a métodos de evaluación confiables y válidos para la valoración del consumo máximo de oxígeno $\left(\mathrm{VO}_{2 \max }\right)$, variable de interés, por ser el indicador más importante para evaluar la condición cardiovascular en la práctica deportiva. La prueba de esfuerzo en banda sin fin, es utilizada frecuentemente para estimar el $\mathrm{VO}_{2 \max }$. Si bien se trata de una prueba segura, representa un esfuerzo físico significativo para el individuo, al punto que solo aquellos deportistas altamente entrenados tienen la capacidad de alcanzar el $\mathrm{VO}_{2 \max }$; además, demanda tiempo y recursos relativamente especializados y costosos.

Por otra parte, se ha generalizado el uso de monitores de frecuencia cardiaca para el control y seguimiento de la intensidad del ejercicio físico en los programas de prescripción, desconociéndose el uso que tienen algunos de ellos, como el Polar $5810 \AA$, para realizar estimaciones del $\mathrm{VO}_{2 \max }$ mediante ecuaciones programadas con datos provenientes del individuo, aún en situaciones de reposo. Sin embargo, no hay suficiente evidencia de que este tipo de estimaciones den cuenta de la potencia aeróbica real de los individuos y que sus resultados sean intercambiables con los obtenidos mediante una prueba de esfuerzo. El presente 
estudio buscó establecer la concordancia existente en la estimación del $\mathrm{VO}_{2 \max }$ entre una prueba de esfuerzo en banda sin fin y el Polar $S 810 \circledR$, en deportistas universitarios de diferentes disciplinas de tipo aeróbico a la altura de Bogotá.

\section{MATERIALES Y METODOS}

A través de la División de Deportes de la Universidad Nacional, se convocó un grupo de estudiantes que cumpliera con los siguientes criterios: ser deportistas mayores de 18 años, de cualquier género, que estuvieran practicando un deporte aeróbico en forma continua durante los últimos seis meses, mínimo 3 veces a la semana, entre 1 y 2 horas; que se encontraran viviendo a la altura de Bogotá; que contaran con una valoración médica previa y fueran declarados aptos para la realización de la prueba de esfuerzo; que tuvieran un índice de masa corporal entre 18,5 y 25 y con un porcentaje de grasa menor del $17 \%$ para hombres y del $25 \%$ para mujeres. La muestra estuvo constituida por una serie consecutiva de 46 voluntarios universitarios. Todos los sujetos fueron informados sobre los riesgos potenciales de este estudio y dieron su consentimiento de forma escrita de acuerdo a las recomendaciones de la normatividad nacional e internacional relacionada con este tipo de investigaciones. El estudio fue avalado por el Comité de Ética de la Facultad de Medicina de la Universidad Nacional de Colombia.

Diseño del estudio

Se diseñó un estudio observacional de concordancia entre dos pruebas que estimaron simultáneamente y de manera enmascarada e independiente el $\mathrm{VO}_{2 \text { máx }}$ en el grupo de deportistas. A los voluntarios se les dio información relacionada con las características de cada una de las pruebas, así como de las condiciones estándares que debían cumplir para la realización de la prueba de esfuerzo. Se registraron datos antropométricos y de hábitos de ejercicio físico. En primera instancia se hizo la estimación mediante el uso del Polar $S 810 \circledR$ y y continuación, se realizó la prueba de esfuerzo en banda sin fin. Los datos obtenidos en el Polar $S 810 \circledR$ fueron registrados por un investigador independiente de aquel que a continuación hizo lo propio con la prueba de esfuerzo. Estos datos no fueron conocidos ni por los deportistas ni por el investigador que realizó la prueba de esfuerzo.

El Polar $S 810 ®$ utiliza una ecuación de predicción en reposo basado en la información del usuario: peso, talla, género, nivel de actividad física y frecuencia cardiaca en reposo. Los participantes definieron su nivel de actividad física como "entrenado" (3). Los datos antropométricos se tomaron con el bioimpedanciómetro 
Tánita TBF300 y los resultados obtenidos se introdujeron en el Polar $S 810 \circledR$ para cada individuo, junto con los del nivel de actividad física declarado.

A cada sujeto se le ubicó el sensor del Polar $S 810$ y se le pidió permanecer en reposo en posición supina durante 15 minutos antes de la medición. Luego, se registró la frecuencia cardiaca y respiratoria de reposo y el dato del $\mathrm{VO}_{2 \max }$ reportado por el monitor de frecuencia cardiaca. Posteriormente, un segundo grupo evaluador entrenado y certificado, aplicó la prueba de esfuerzo a cada individuo, la cual se realizó en una banda sin fin Quinton $Q 710$, siguiendo el protocolo de Balke (4). Tanto en mujeres como en hombres, la prueba se culminó cuando la persona manifestó un alto nivel de fatiga (puntuación de 20 según la escala de Borg) (5) y/o cuando no se presentaron cambios en la frecuencia cardiaca a pesar del incremento en la carga de trabajo, lo que se asumió como indicador de que el sujeto había alcanzado su $\mathrm{VO}_{2 \max }$. En el transcurso de la prueba se tomaron datos de las variables fisiológicas como frecuencia cardiaca y presión arterial, para controlar cualquier riesgo. Posteriormente, se calculó el $\mathrm{VO}_{2 \max }$, según la fórmula propuesta para el protocolo aplicado (6).

\section{Análisis estadístico}

La población se describió mediante medidas de tendencia central y dispersión; igual se procedió para el análisis de los datos del $\mathrm{VO}_{2 \max }$. Se examinó la normalidad mediante la prueba de Shapiro Wilks. Previo a valorar la concordancia entre los dos métodos, se hizo la prueba de homocedasticidad de los valores de $\mathrm{VO}_{2 \max }$ mediante el test de Levene (7), encontrándose que no eran homocedásticos, lo que llevó a verificar una normalización de las distribuciones mediante el logaritmo de los mismos.

La determinación de la concordancia entre los datos de $\mathrm{VO}_{2 \max }$ por los dos métodos, fue establecida a través del Coeficiente de Correlación Concordancia de Lin (CCC) (8). Para los propósitos de este estudio y en razón a que no hay referentes previos, se supuso razonable desde el punto de vista fisiológico, que fuera tolerable para hallar una "buena" concordancia, encontrar diferencias hasta de $1,5 \mathrm{~mL} / \mathrm{Kg} / \mathrm{min}$ y que los límites de acuerdo no superaran el $+/-5 \%$; se asumió que la concordancia sería "aceptable" para diferencias de hasta $2 \mathrm{~mL} /$ $\mathrm{Kg} / \mathrm{min}$ y que no superaran los límites de acuerdo más allá de $+/-7,5 \%$ y se calificó como no tolerables, diferencias entre los valores superiores a $5 \mathrm{~mL} / \mathrm{Kg} /$ min y que superaran los límites de acuerdo más allá del $10 \%$. Los datos se registraron en una base de datos en el programa Acces y los valores relevantes fueron transferidos y analizados con Stata $8 \circledR$. 


\section{RESULTADOS}

Se valoraron 46 deportistas, 15 mujeres y 31 hombres (edad: $23,6 \pm 3,8 ; 22,7$ años, respectivamente; talla: $1,67 \pm 0,1 \mathrm{~m}$; peso: $60,8 \pm 8,3 \mathrm{Kg}$; IMC: $21,5 \pm 1,8 \mathrm{Kg} / \mathrm{m}^{2}$ ); 27 individuos realizaban prácticas de carácter individual. El 45,7\% de los deportistas jugaba fútbol, el 21,7 \% hacía ciclismo, el $13 \%$ baloncesto, el 6,5 \% natación, igual porcentaje atletismo y el 6,6\% restante, otros deportes. El valor promedio ( \pm DE) de $\mathrm{VO}_{2 \max }$ obtenido mediante el Polar $S 810 ®$ fue de $43,1 \pm 5,4 \mathrm{~mL} / \mathrm{Kg} /$ $\min$ (IC $95 \% 41,5-44,7$ ) y el obtenido a través de la prueba de esfuerzo alcanzó $44,9 \pm 10,8 \mathrm{~mL} / \mathrm{Kg} / \mathrm{min}$ (IC $95 \% 41,7-48,1$ ). Como se puede observar en las Figuras 1 y 2, hay diferencias evidentes en la distribución de tales valores de $\mathrm{VO}_{2 \max }$ : en el caso del Polar S810® hay una tendencia de la mitad de los valores a concentrarse alrededor de un punto medio, con algunos valores extremos, en tanto que para la prueba de esfuerzo se identifica un valor verdaderamente extremo y una distribución de los datos con un rango de mayor amplitud.

\begin{tabular}{|c|c|c|c|}
\hline & $\begin{array}{c}\mathrm{VO}_{2 \max } \\
\text { POLAR } \\
\text { S810® } \\
(\mathrm{mL} / \mathrm{Kg} / \mathrm{min})\end{array}$ & $\begin{array}{c}\mathrm{VO}_{2 \max } \\
\mathrm{MAXIMAL} \\
\text { (mL/Kg/min) }\end{array}$ & Valor de $\mathrm{p}^{*}$ \\
\hline Promedio & 43.1 & 44,9 & 0.0000 \\
\hline $\mathrm{DE}$ & 5.4 & 10,8 & \\
\hline Valor mínimo & 34 & 25,9 & \\
\hline Valor máximo & 62 & 82,7 & \\
\hline Percentil $25-75$ & $40-44$ & $34,2-42,5$ & \\
\hline
\end{tabular}

Al comparar los dos métodos (Tabla 1), se observa que el valor del promedio difiere en $1,8 \mathrm{~mL} / \mathrm{Kg} / \mathrm{min}(\mathrm{p}<0,0000)$. Sin embargo el rango entre el valor mínimo y máximo por el Polar $S 810 \circledR$ es de $28 \mathrm{~mL} / \mathrm{Kg} / \mathrm{min}$, en tanto que mediante la prueba de esfuerzo es del doble $(56,8 \mathrm{~mL} / \mathrm{Kg} / \mathrm{min})$ lo que ratifica las diferencias observadas gráficamente entre sus distribuciones. La concordancia entre los valores obtenidos de VO2max entre el Polar y la prueba de esfuerzo fue del 30,8 $\%$ (CCC de Lin 0,308, IC $95 \% 0.104-0.512, \mathrm{p}=0,003$ ), que corresponde a una pobre correlación de concordancia. Esta no mejoró al hacer el análisis por subgrupos, así: para el género masculino el CCC fue 0,29 (IC $95 \%$ 0,04-0,54); para las mujeres, alcanzó un CCC 0,15 (IC 95\% -0,14-0,45); el análisis por tipo de deporte arroja un CCC grupal de 0,38 (IC 95 \% 0,04-0,54) e individual de 0,16 (IC $95 \%-0,20-0,52$ ) y tan solo en los deportistas entre 24,1 y 28 años de edad mejoró su valor (CCC 0,65 IC 95 \% 0,35-0,93). La concordancia no se modificó 
sustancialmente al hacer la normalización mediante la función logaritmo de las distribuciones de los valores de $\mathrm{VO}_{2 \max }$ obteniéndose un CCC de 0,313 (IC $95 \%$ $0,101-0,525, \mathrm{p}<0,004)$.

Figura 1. $\mathrm{VO}_{2 \max }$ mediante el uso del Polar $\mathrm{S} 810^{\mathrm{B}}$ y mediante la prueba de esfuerzo
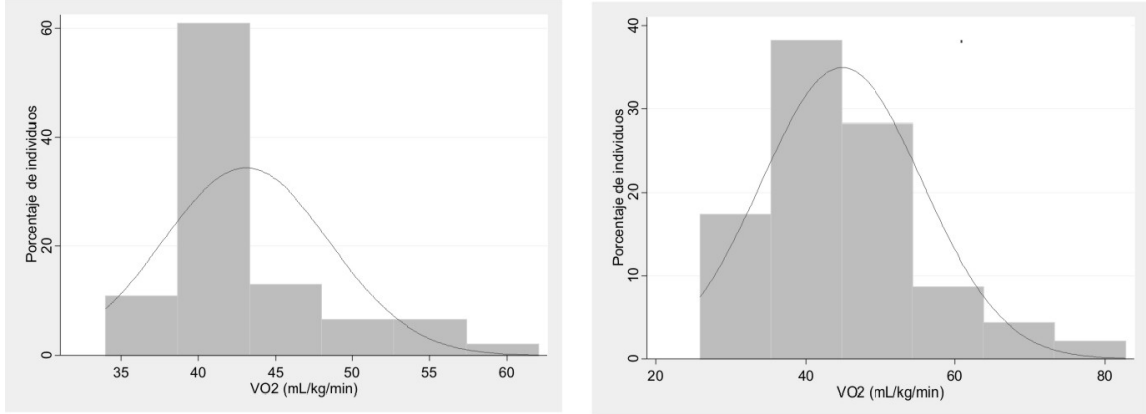

Figura 2. Estimación del $\mathrm{VO}_{2 \max }(\mathrm{mL} / \mathrm{Kg} / \mathrm{min})$ mediante dos métodos

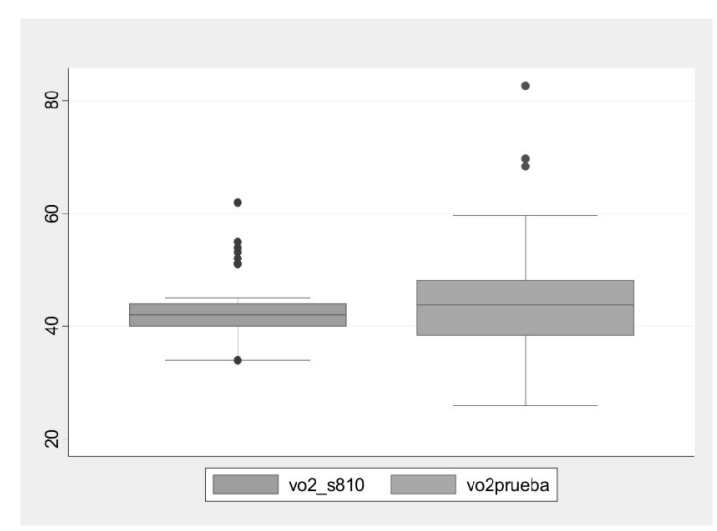

La Figura 3 presenta la relación cartesiana entre los valores del $\mathrm{VO}_{2 \max }$, obtenidos por los dos métodos. De encontrar perfecta concordancia entre ellos, se esperaría que la nube puntos estuviese siguiendo la línea de $45^{\circ}$ allí marcada. Como se observa, tal línea cruza transversalmente la línea de perfecta concordancia con valores concurrentes alrededor de $42 \mathrm{~mL} / \mathrm{Kg} / \mathrm{min}$ de prueba de esfuerzo; para datos inferiores a este valor, el Polar $5810 \circledR$ arroja datos numéricos superiores y para valores superiores hace lo contrario, tendencia que se marca, en la medida en que se hacen más grandes los valores observados por la prueba de esfuerzo. La gráfica de Bland y Altman (Figura 4) y la determinación 
de los límites de acuerdo, establece que los límites de las dos desviaciones estándar alrededor de la puntuación promedio de las diferencias entre las dos mediciones son muy amplios: varían entre $-21,4$ y $17,8 \mathrm{~mL} / \mathrm{Kg} / \mathrm{min}$. Tal comportamiento ratifica la pobre concordancia entre los dos métodos.

Figura 3. Dispersión de los valores del $\mathrm{VO}_{2 \max }$ entre el Polar $S 810 \AA$ y una prueba de esfuerzo

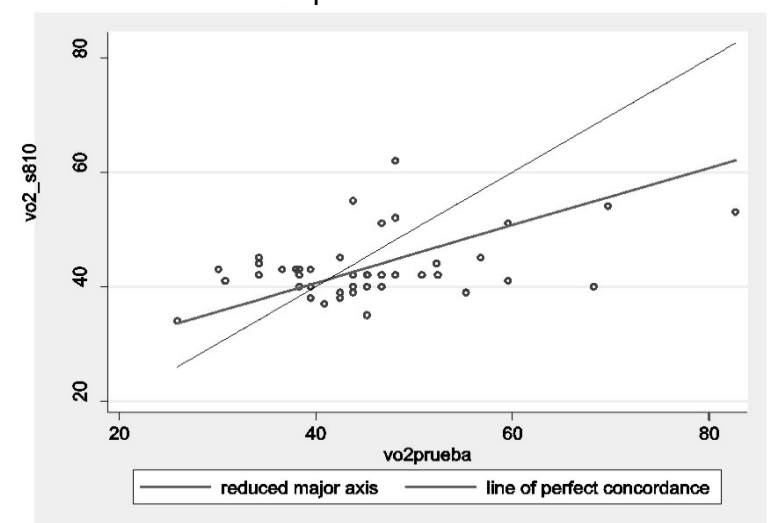

Figura 4. Concordancia en la estimación del $\mathrm{VO}_{2 \max }$ entre el Polar $S 810 \AA$ y una prueba de esfuerzo.

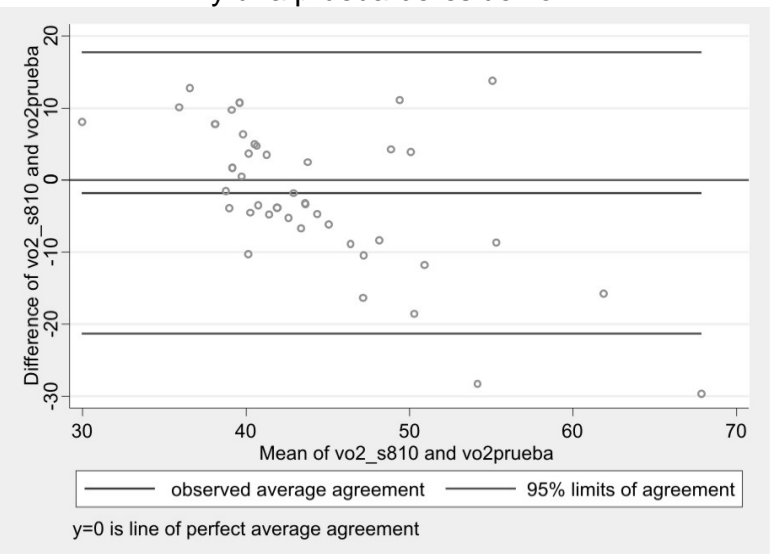

Esta investigación es la primera que, de acuerdo con la búsqueda de literatura científica realizada, aborda como objeto de estudio, el análisis de la correlaciónconcordancia entre dos formas de estimación del $\mathrm{VO}_{2 \max }$, como un indicador proxy del rendimiento físico a saber: la tradicional prueba de esfuerzo, frente a una prueba en reposo mediante el uso del Polar S810®. Se encontró una pobre concordancia entre el Polar $S 810 \circledR$ y la prueba de esfuerzo según el protocolo 
de Balke. No son muchos los estudios descritos que, usando el Polar S810®, indaguen acerca de su uso y los que lo hacen, examinan otras utilidades (9-12). Sin embargo, los métodos explorados aquí, hacen una estimación indirecta del $\mathrm{VO}_{2 \max }$, por lo que en ausencia de mediciones ergoespirométricas, no es posible conocer cuál de ellos aporta una medida más fiable.

Hubo heterogeneidad en la disciplina deportiva practicada, así como en la duración e intensidad del entrenamiento, lo que podría explicar la variabilidad encontrada en los valores obtenidos de $\mathrm{VO}_{2 \max }$. Existe información acerca de que en deportistas de diversas disciplinas de alto nivel de entrenamiento aeróbico, se alcanzan valores de $\mathrm{VO}_{2 \max }$ diferentes (13-15); Saltin refiere en corredores de media distancia, valores de $\mathrm{VO}_{2 \max }$ de $80 \mathrm{~mL} / \mathrm{Kg} / \mathrm{min}$; para ciclistas, de 75 $\mathrm{mL} / \mathrm{Kg} / \mathrm{min}$ y en remeros, de $61 \mathrm{~mL} / \mathrm{Kg} / \mathrm{min}$ (16). Por otro lado, las posibles diferencias puedan deberse a que cada especialidad o modalidad deportiva tiene un patrón cine-antropométrico diferente y por tanto existe un claro prototipo físico para alcanzar el rendimiento deportivo correspondiente, lo cual determina diferencias en el $\mathrm{VO}_{2 \max }$. Igual situación acontece con el rendimiento cuando la práctica es individual o colectiva (17-19). En este sentido, podría explicarse la poca concordancia encontrada entre las dos pruebas, por factores no contemplados por el Polar S810® como la disciplina deportiva practicada.

Hasta donde se conoce, los cálculos obtenidos a través del Polar S810®, no consideran el concepto de altura sobre el nivel del mar a la cual se realiza la valoración y ello se pueda convertir en un factor de confusión a la hora del cálculo, que incluso eventualmente, pudiera explicar la pobre concordancia encontrada. Otros estudios que consideren esta circunstancia, deben realizarse a fin de determinar posibles explicaciones al respecto.

El bajo valor del coeficiente de Lin, no autoriza entonces, intercambiar la estimación del $\mathrm{VO}_{2 \max }$ derivada del Polar $S 810 \circledR$, por el valor proveniente de la prueba de esfuerzo hecha con la prueba de esfuerzo utilizando el protocolo de Balke.

La normalización de la distribución hallada en este estudio para los valores estimados y la subsiguiente aplicación de la concordancia, no permitió observar una modificación sustancial del dato inicialmente hallado, lo que pareciera significar que el coeficiente de correlación y concordancia de Lin no se modifica en términos de la distribución normal de la variable. Sin embargo, esta hipótesis desborda el alcance del presente estudio y deberá someterse a futuro análisis. Dados los 
hallazgos exploratorios realizados, es muy probable que, al menos a la altura de Bogotá y con el biotipo de deportistas estudiados, sean necesarios ajustes lineales significativos a los valores encontrados en el Polar S810®, mediante modelos matemáticos de predicción, con los que se asegure concordancia y se propicie intercambiabilidad, entre la prueba de esfuerzo y el Polar S810®, en la estimación del $\mathrm{VO}_{2 \max }$, como indicador proxy del acondicionamiento físico deportivo *

\section{REFERENCIAS}

1. American College of Sports Medicine. ACSM's Guidelines for exercise Testing and prescription. Ed Lippincott Williams \& Wilkins. 7th ED; 2005.

2. Congreso de Colombia [Internet]. Disponible en http://www.redcreacion.org/documentos/ ley181.htm. Consultado en noviembre de 2008.

3. Tanaka H, Monahan K, Seals D. Age-Predicted Maximal Heart Rate Revisited. J of Am Col Card 2011; 37(1) 153-156.

4. Marinov B, Kostianev S, Turnovska T. Modified treadmill protocol for evaluation of physical fitness in pediatric age group-comparasion with Bruce and Balke protocols. Acta Physiol Pharmal Bulg 2003;27(2-3):47-51.

5. Mador MJ, Rodis A, Magalang UJ. Reproducibility of Borg scale measurements of dyspnea during exercise in patients with COPD. Chest 1995;107:1590-97.

6. Heyward, Vivian H. Advanced fitness assessment and exercise prescription. 5th Ed. Human Kinetics; 2006.

7. O'Brien P. C. Robust procedures for testing equality of covariance matrices Biometrics. 1992; 48 (3): 819-827.

8. Lin L. A concordance correlation coefficient to evaluate reproducibility. Biometrics 1989; 45:255268.

9. Shvartz E, Reibold RC. Aerobic fitness norms for males and females aged 6 to 65 years: a review. Aviat Space Environ Med. 1990 ; 61(1): 3-11.

10. Wierzbicka-Damska I, Samolyk A, Jethon Z et al. Physical efficiency of 10-16 years old boys with hearing impairment. Ann Academiae Med Bial. 2005; 50(1):167-169.

11. Kingsley M, Lewis MJ, Marson RE. Comparisson of Polar S810 and an Ambulatory ECG System for RR Interval Measurement during progressive exercise. Int J Sports Med 2005; 26:3944.

12. Gamelin FX, Berthoin S, Bosquet L. Validity of the Polar S810 Heart rate Monitor to measure RR intervals at rest. Med. Sci. Sports Exerc. 2006; 38(5):887-893.

13. Holmér I. Oxygen uptake during swimming in man. J Appl Physiol. 1972;33(4):502-509.

14. Kemi OJ, Hoff J, Engen LC, Helgerud J, Wisløff U. Soccer specific testing of maximal oxy-gen uptake. J Sports Med Phys Fitness. 2003; 43(2):139-144.

15. Aziz AR, Chia M, Teh KC. The relationship between maximal oxygen uptake and repeated sprint performance indices in field hockey and soccer players. J Sports Med Phys Fitness 2000 Sep; 40(3):195-200.

16. Saltin B, Astrand PO. Maximal oxygen uptake in athletes.J Appl Physiol. 1967; 23(3):353358.

17. Cabañero M, Canda A, Bonilla M, Calderón $C$, Rubio S. Gimnasia rítmica de alta competi-ción: composición corporal y somatotipo. Archivos de Medicina del Deporte. XVI, Especial 1999; 505-506.

18. Zabukovec R, Tiidus PM. Physiological and anthropometric profile of elite kickboxers. Jour-nal of strength and conditioning research 1995; 9(4): 240-242. 